\title{
Absolute spectral modelling of asteroid (4) Vesta
}

\section{Martikainen, Julia}

2019-02

Martikainen , J , Penttilä , A , Gritsevich , M , Videen , G \& Muinonen , K O 2019 , ' Absolute spectral modelling of asteroid (4) Vesta ', Monthly Notices of the Royal Astronomical Society pÿ, vol. 483 , no. 2 , pp. 19521956 . https://doi.org/10.1093/mnras/sty3164

http://hdl.handle.net/10138/300975

https://doi.org/10.1093/mnras/sty3164

cc_by_nc_sa

publishedVersion

Downloaded from Helda, University of Helsinki institutional repository.

This is an electronic reprint of the original article.

This reprint may differ from the original in pagination and typographic detail.

Please cite the original version. 


\title{
Absolute spectral modelling of asteroid (4) Vesta
}

\author{
Julia Martikainen, ${ }^{1 \star}$ Antti Penttilä, ${ }^{1}$ Maria Gritsevich, ${ }^{1,2}$ Gorden Videen ${ }^{3}$ and \\ Karri Muinonen ${ }^{1,4}$ \\ ${ }^{1}$ Department of Physics, University of Helsinki, PO Box 64, FI-00014 Helsinki, Finland \\ ${ }^{2}$ Institute of Physics and Technology, Ural Federal University, Mira str 19, 620002 Ekaterinburg, Russia \\ ${ }^{3}$ Space Science Institute, 4750 Walnut St, Suite 205 Boulder, CO 80301, USA \\ ${ }^{4}$ Finnish Geospatial Research Institute FGI, PO Box 84, FI-00521 Helsinki, Finland
}

Accepted 2018 November 17. Received 2018 November 8; in original form 2018 February 9

\begin{abstract}
We present a new physics-based approach to model the absolute reflectance spectra of asteroid (4) Vesta. The spectral models are derived by utilizing a ray-optics code that simulates light scattering by particles large compared to the wavelength of the incident light. In the light of the spectral data obtained by the Dawn spacecraft, we use howardite powder to model Vesta's surface regolith and its particle size distribution for 10-200 $\mu \mathrm{m}$ sized particles. Our results show that the modelled spectrum mimics well the observations. The best match was found using a power-law particle size distribution with an index 3.2. This suggests that Vesta's regolith is dominated by howardite particles $<25 \mu \mathrm{m}$ in size.
\end{abstract}

Key words: scattering - methods: numerical - techniques: spectroscopic-minor planets, asteroids: individual: Vesta.

\section{INTRODUCTION}

Vesta is the second largest object in the asteroid belt. Unlike its distant icy neighbour Ceres, Vesta is dense and rocky, with evidence of massive past collisions on its surface. These collisions spewed debris throughout the solar system and are the source event for Vestoids (Reddy, Nathues \& Gaffey 2011). Some of this debris has been collected on the Earth in the form of howardite, eucrite, and diogenite (HED) meteorites (McCord, Adams \& Johnson 1970; McSween et al. 2013). These meteorites are an important source of information about the Vesta regolith.

Vesta is currently the only known differentiated asteroid. The Dawn probe found it has a basaltic crust and a metallic core with a diameter above $200 \mathrm{~km}$ (De Sanctis et al. 2012; Reddy et al. 2012; Russell et al. 2012). Ground-based observations showed photometric variations that were believed to be caused by a hemispheric-scale 'albedo spot' (see Cellino et al. 1987; Cellino et al. 2016) that was interpreted to correspond to a large impact crater. The Hubble Space Telescope images (Thomas et al. 1997) showed evidence of a large impact basin on Vesta's Southern hemisphere. These observations were confirmed by Dawn that found two enormous impact craters, Rheasilvia and Veneneia, near Vesta's south pole (Jaumann et al. 2012). Rheasilvia is about 1 Gyr old (Marchi et al. 2012) and has a diameter of $500 \pm 25 \mathrm{~km}$ (Schenk et al. 2012). The older of the two craters, Veneneia, is about 2 Gyr old with a diameter of $400 \pm 25 \mathrm{~km}$ (Schenk et al. 2012). Veneneia has been partly destroyed by Rheasilvia.

^E-mail: julia.martikainen@ helsinki.fi
A photometric phase curve describes the brightness of an asteroid as a function of phase angle, which is the angle between the Sun and the observer as seen on the asteroid. The phase curve of Vesta shows that the reflectivity decreases, as is common, with increasing phase angle (e.g. Hicks et al. 2014). In addition, the reflectance spectrum depends on the phase angle. These spectral phase effects add their own contribution to the observed spectra: the absorption band depth increases, and the spectral slope reddens as the phase angle increases. Reddy et al. (2012) studied spectral phase effects on Vesta's spectrum and found that the spectral slope in the visible increased with increasing phase angle. In the NIR, the Band Area Ratio (the ratio of the absorption band area at around $2.0 \mu \mathrm{m}$ to the absorption band area centred at $0.95 \mu \mathrm{m}$ ) and the band depths increased when the phase angle increased.

Multiple ground-based spectroscopic observations of Vesta have been carried out over the years. To mention a few, Chapman, Gaffey \& McFadden (1993) observed the reflectance spectra of 285 asteroids, including Vesta, over the wavelength region of 0.32 $1.08 \mu \mathrm{m}$. Busarev (2010) used a $1.25 \mathrm{~m}$ telescope to obtain Vesta's spectrum at $0.39-0.91 \mu \mathrm{m}$ region. Vesta's spectrum has also been observed in the mid-IR region by the ISO telescope with the ISOPHOT instrument (Dotto et al. 2000), the Palomar observatory (Lim et al. 2005), and the Kuiper Airborne Observatory (KAO) (Cohen et al. 1998). In this paper, we use the reflectance spectra observed by Reddy (2011) at a wavelength range of $0.7-2.5 \mu \mathrm{m}$ with a phase angle of $17.4^{\circ}$.

In 2011, the Dawn spacecraft began a 1 yr survey of Vesta with the Visible and Infrared Spectrometer (VIR) and the Dawn Framing Camera. The VIR has two channels. The visible channel covers 
Table 1. The relative rotational phases of the selected observed Vesta spectra. Each spectrum number refers to a file name (vestaXX.tab) in the observational data set.

\begin{tabular}{lccc}
\hline Spectrum & Rotational phase & Spectrum & Rotational phase \\
\hline 01 & 0.00 & 07 & 0.30 \\
02 & 0.05 & 18 & 0.86 \\
03 & 0.09 & 19 & 0.92 \\
04 & 0.13 & 20 & 1.00 \\
05 & 0.16 & 21 & 1.05 \\
06 & 0.20 & 22 & 1.09 \\
\hline
\end{tabular}

the wavelength range from 0.25 to $1 \mu \mathrm{m}$ with a spectral resolution of $1.89 \mathrm{~nm}$ and a field of view of $64 \mathrm{mrad}$. The infrared channel covers the wavelength range from 1 to $5 \mu \mathrm{m}$ with an average spectral resolution of $9.4 \mathrm{~nm}$ (De Sanctis et al. 2011). The spectral range incorporates numerous ice and mineral spectral features, and is designed to provide information about the surface components of Ceres and Vesta. While outfitted aboard the orbiting Dawn spacecraft, this instrument provides these spectral components as a function of phase angle, which can be used to ascertain information on the regolith morphology. The Dawn Framing Camera collected multispectral, high-resolution data in the visible wavelength region (Reddy et al. 2012). This data was used to obtain the global photometric properties of Vesta (Li et al. 2013). Furthermore, the clear filter images were analysed by Schröder et al. (2014) to retrieve a photometric characterization of Vesta's surface in unprecedented detail. In the future, we plan to study the data taken by Dawn.

The reflectance spectra of asteroids have been interpreted by numerous laboratory studies and empirical models, such as the Hapke model (Hapke 1993), but never before using physics-based light scattering codes. We have recently developed a new simulation framework (Markkanen et al. 2018; Muinonen et al. 2018) to model the absolute reflectance spectra of planetary materials. This approach was used to model the absolute reflectance spectra of meteorites (Martikainen et al. 2018). In this paper, we use a howardite sample to model the absolute reflectance spectra of Vesta and to obtain the size distribution for the regolith particles on Vesta's surface.

\section{MEASUREMENTS AND DATA}

\subsection{Spectral measurements}

The data provided by the Dawn mission (De Sanctis et al. 2013) suggest that the mineral composition of Vesta's surface regolith is similar to howardites, achondritic stony meteorites that together with eucrites and diogenites represent the HED meteorite clan. We utilized the University of Helsinki UV-vis-NIR integrating-sphere spectrometer (Penttilä et al. 2018) to measure the reflectance spectrum of howardite powder (Kohout et al. 2015) using a wavelength range of $0.25-3.2 \mu \mathrm{m}$ with a $5 \mathrm{~nm}$ resolution. The particle sizes of the howardite powder ranged from 50 to $100 \mu \mathrm{m}$.

\subsection{Observational data}

We utilized the reflectance spectra of Vesta observed by Reddy (2011) with the SpeX instrument on NASA Infrared Telescope Facility (IRTF) on Mauna Kea, Hawaii. The data set contains Vesta's normalized reflectance spectra observed at a $17.4^{\circ}$ phase angle during different rotational phases. We selected 12 of the normalized spectra (Table 1) that were not affected by Vesta's albedo spot (see Section 1) and further scaled them to a geometric albedo value of

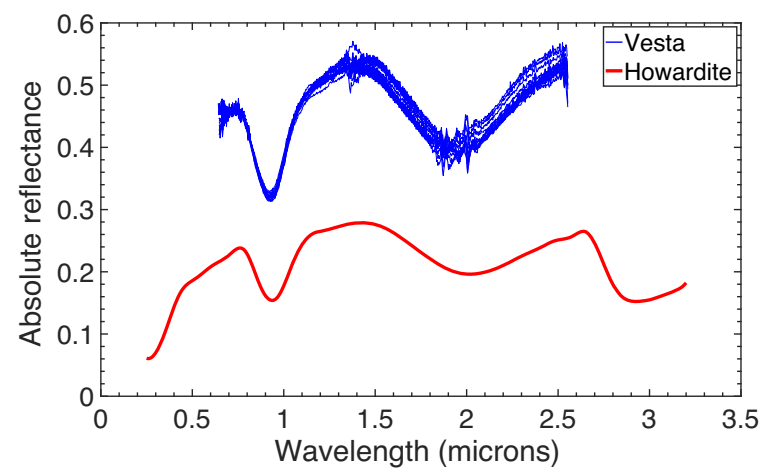

Figure 1. The observed spectra of Vesta and the measured spectrum of howardite.

0.423 (Tedesco et al. 2004) at $0.55 \mu \mathrm{m}$. Fig. 1 shows the spectra of Vesta together with that of the measured howardite sample.

\section{NUMERICAL METHODS}

\subsection{Absolute spectral modelling}

Asteroids consist of different minerals, each of them having their own dominant spectral features. The combinations of the minerals cause changes to their spectra, such as relocation of the minima, modification in the depth of the absorption bands and in the intensity level of the spectrum. The absolute reflectance spectra of asteroids can be modelled by using the most common minerals that dominate their spectral features. Due to the knowledge that the regolith on Vesta's surface consists mostly of howardite (see Section 2.1), we used this material in our computations.

Absolute spectral modelling from 0.4 to $2.5 \mu \mathrm{m}$ was carried out by utilizing the SIRIS4 ${ }^{1}$ (Lindqvist et al. 2018; Martikainen et al. 2018, see also Muinonen et al. 2009) code that simulates light scattering by Gaussian-random-sphere particles (see Muinonen et al. 2009 and Martikainen et al. 2018) larger than the wavelength of the incident light. The code accounts for inhomogeneous waves in absorbing material, which can include small, diffuse scatterers. SIRIS4 is available at. For the computations, SIRIS4 requires the complex refractive index $m$ of the material and the particle shape as input. The complex refractive index as a function of the wavelength for howardite was obtained by using an optimization code (Martikainen et al. 2018) that utilizes SIRIS4 and the measured reflectance spectrum of the material (see Section 3.2).

Using the derived complex refractive index, we computed the scattering matrices and single-scattering albedos for howardite particles with sizes (diameters) ranging from 10 to $200 \mu \mathrm{m}$ in steps of 10 $\mu \mathrm{m}$. The obtained scattering matrices and single-scattering albedos were then averaged over a power-law distribution for three different size ranges: $10-50,10-100$, and 10-200 $\mu \mathrm{m}$. The power-law index $v$ used for averaging the scattering matrices and single-scattering albedos was selected so that the final modelled spectrum produced the best match with the observed spectra. Averaging was carried out using the method presented in Martikainen et al. (2018). The final spectrum was computed using the averaged howardite particles as diffuse scatterers inside a Vesta-sized volume.

${ }^{1}$ SIRIS4, 2018, University of Helsinki Planetary System Research group home page, SIRIS4. Available at: https://wiki.helsinki.fi/display/PSR. 


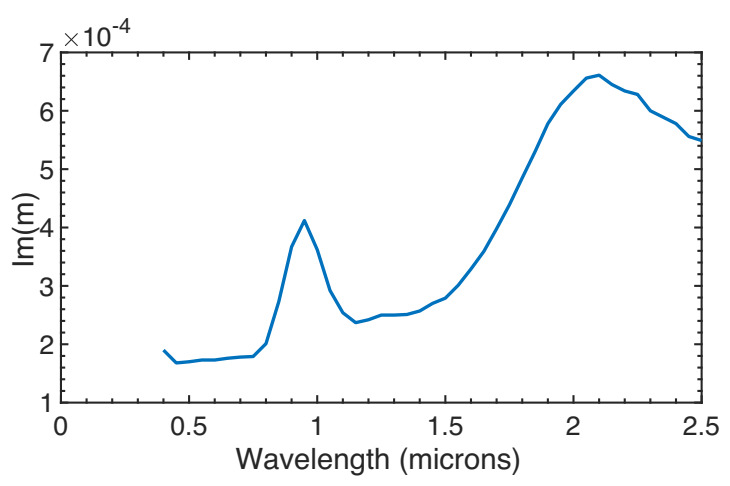

Figure 2. The derived imaginary part of the refractive index for howardite powder with a $60 \mu \mathrm{m}$ grain size.

Asteroid surfaces are rough and porous, and there is shadowing among the regolith particles. The roughness on the regolith surface-element scale can also cause (self-)shadowing (Wilkman, Muinonen \& Peltoniemi 2015; Wilkman et al. 2016). We modelled the spectra at a $17.4^{\circ}$ phase angle. Since a spectrum taken at a phase angle of $17.4^{\circ}$ is considered, and shadowing can therefore play a role, some normalization taking into account how much the brightness decreases coming from solar opposition to $17.4^{\circ}$ is necessary. Therefore, we studied the photometric phase curve of Vesta (Hicks et al. 2014) that, not unexpectedly, shows non-linear opposition brightening. When going from the opposition to a $17.4^{\circ}$ phase angle, Vesta's reflectance decreases by a factor of 0.491 at 0.55 $\mu \mathrm{m}$. We used this factor on Vesta's spectrum that was scaled to a geometric albedo at a zero phase angle (see Section 2.2) in order to get back to $17.4^{\circ}$ and account for the spectral phase effects (see Section 1). Thereafter, the entire spectrum can be considered to be absolute across the whole wavelength range.

\subsection{Derivation of the refractive index}

We derived the imaginary part of the refractive index by utilizing an optimization code presented in Martikainen et al. (2018). Howardite's spectrum is dominated by the strong spectral features of pyroxene and thus the real part of $m$ was fixed according to the refractive index of pyroxene. For the real part of $m$, we used 1.8 at all wavelengths because it does not change significantly over the modelled wavelength region (Scott \& Duley 1996). We used a particle size of $60 \mu \mathrm{m}$ for the howardite powder. The derived $\operatorname{Im}(m)$ is shown in Fig. 2.

A sensitivity analysis was carried out for the derived complex refractive indices. Since the particle sizes of the measured howardite powder range from 50 to $100 \mu \mathrm{m}$, we used the derived refractive indices to compute the spectrum of howardite powder with a Gaussian distribution for a size range of 50-100 $\mu \mathrm{m}$ using a standard deviation of 5 and a mean of $60 \mu \mathrm{m}$. The computed spectrum was then compared with the spectrum that was obtained using a particle size of $60 \mu \mathrm{m}$ (see Fig. 3). The resulting spectra show that the size distribution does not change the spectrum significantly, and thus we can utilize the derived refractive indices of howardite powder with a $60 \mu \mathrm{m}$ grain size.

The next step was to study how the shapes of the Gaussianrandom-sphere particles used in SIRIS4 affect the derived $\operatorname{Im}(m)$. First, we used parameter $v_{\mathrm{G}}$ values 2.5 and 3.5 for the autocorrelation function of the logarithmic radial distance. Secondly, we set the standard deviation of the particle radius $\sigma_{\mathrm{G}}$ to 0.12 and 0.22 .

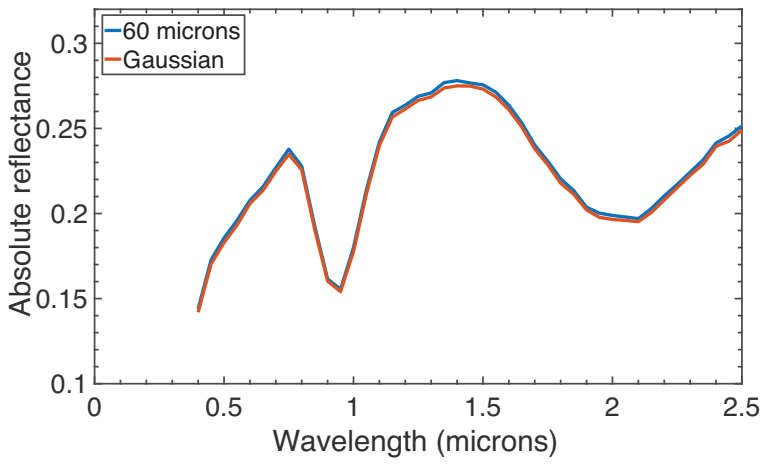

Figure 3. The reflectance spectra of howardite powder with a Gaussian size distribution and a $60 \mu \mathrm{m}$ grain size.
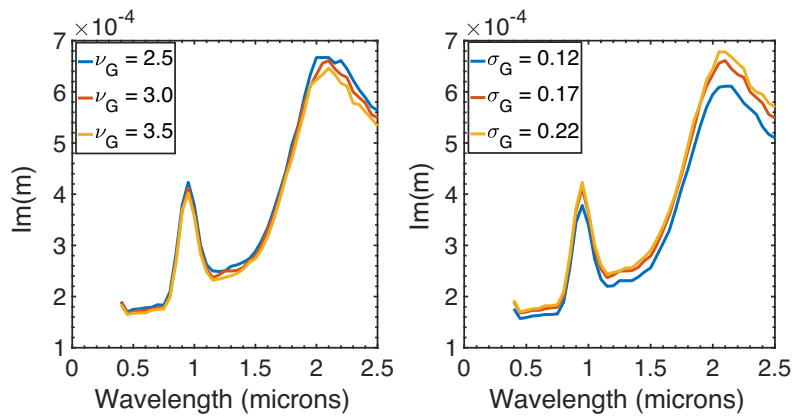

Figure 4. The derived imaginary parts of the refractive indices using different $\nu_{\mathrm{G}}$ and $\sigma_{\mathrm{G}}$.

We then compared the derived $\operatorname{Im}(m)$ with the derived values used in our spectral models where we set $v_{\mathrm{G}}$ to 3.0 and $\sigma_{\mathrm{G}}$ to 0.17 (see Fig. 4).

Changing the $v_{\mathrm{G}}$ for the Gaussian spheres does not affect the derived $\operatorname{Im}(m)$ remarkably because the mean particle volume is independent of $v_{\mathrm{G}}$. However, $\sigma_{\mathrm{G}}$ has a small effect on the derived values. Larger $\sigma_{\mathrm{G}}$ produces larger values for $\operatorname{Im}(m)$, which is due to the slight variations of the phase functions for the Gaussian spheres. The differences increase when going towards longer wavelengths.

\section{RESULTS AND DISCUSSION}

The SIRIS4 light-scattering code was run for the wavelength region of 0.4-2.5 $\mu \mathrm{m}$ with $0.05 \mu \mathrm{m}$ steps to model the absolute reflectance spectrum of Vesta. The detailed description of the computational steps is presented in Section 3.1. A single run took around $2 \mathrm{~h}$ on a single CPU and the computations for all of the wavelengths were run in parallel. The number of sample particles used for each run was 1000 and the number of rays was 2000000 .

Fig. 5 shows the absolute spectrum of Vesta and the modelled absolute spectra using different size ranges and power-law indices at a $17.4^{\circ}$ phase angle. We modelled size ranges of $10-50 \mu \mathrm{m}$ and $10-100 \mu \mathrm{m}$ to demonstrate how the upper particle size limit affects the modelled spectrum; however, the size range from 10 to $200 \mu \mathrm{m}$ is more realistic, assuming asteroid surfaces also contain particles that are larger than $100 \mu \mathrm{m}$. The best match between the modelled spectrum and the observed Vesta spectra for a size range of $10-200 \mu \mathrm{m}$ was found by using $v=3.2$. Changing the upper size limit changed the modelled spectrum significantly: for a size range of $10-50 \mu \mathrm{m}$, the best match was found using $v=1.8$, and for $10-100 \mu \mathrm{m}$, we used $v=2.8$. The results suggest that Vesta's 


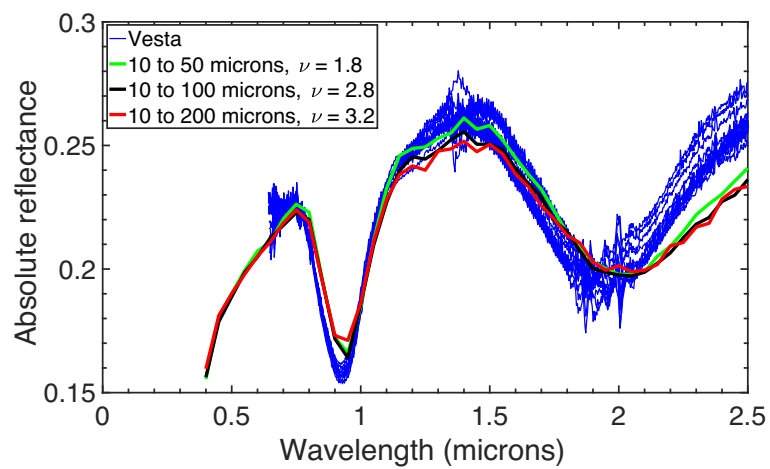

Figure 5. The observed spectra of Vesta and the modelled spectra with different size ranges and power-law indices at a $17.4^{\circ}$ phase angle.
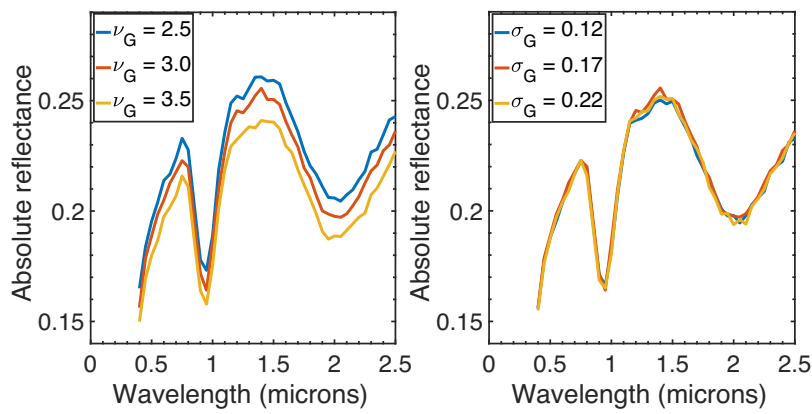

Figure 6. The modelled absolute reflectance spectra of Vesta for a particle size range of $10-100 \mu \mathrm{m}$ using different $v_{\mathrm{G}}$ and $\sigma_{\mathrm{G}}$.

surface regolith is dominated by small howardite particles (more than 75 percent of particles with a grain size $<25 \mu \mathrm{m}$ ), which is consistent with earlier work by Hiroi, Pieters \& Takeda (1994).

There are a few noticeable differences between the modelled spectrum and the observed spectra: the band depths of the modelled spectrum are too shallow, and the centres of the absorption bands are shifted to longer wavelengths. A potential explanation for the lower amplitudes in the spectrum is that we derived too small values for $\operatorname{Im}(m)$ due to omitting wavelength-scale surface-roughness and/or volume-inhomogeneity effects in the $60 \mu \mathrm{m}$ howardite particles when retrieving the refractive indices. By utilizing these small values in the Vesta modelling using pure geometric optics, we end up with too shallow band depths. For the $2-2.5 \mu \mathrm{m}$ wavelength region, the same wavelength-scale factors could be affecting the modelled spectrum; however, the differences can also be caused by the specific composition of our howardite sample, by the presence of other minerals on Vesta's surface that affect the observed spectrum, or by a more elaborate size distribution of particles than the power law. The region at the high end of the considered wavelength interval could also be mildly affected by some contribution from the low-end tail of the thermal emission spectrum. Furthermore, our howardite sample was measured in a room temperature, whereas the observational data were taken at $180-200 \mathrm{~K}$. The study carried out by Reddy et al. (2012) shows that increasing temperature shifts the absorption band centres to longer wavelengths.

Finally, we used the approach described in Section 3.2 to examine how the shapes of the Gaussian-random-sphere particles affect the modelled spectra (see Fig. 6). This sensitivity test was applied to the modelled spectrum with a particle size range of 10-100 $\mu \mathrm{m}$ (averaged over a power-law distribution with $v=2.8$ ). Using different values for $\sigma_{\mathrm{G}}$ does not affect the spectrum significantly, if at all.
This is because the volume has been normalized to the same value for different $\sigma_{\mathrm{G}}$ (Martikainen et al. 2018). The largest differences can be seen when changing $v_{\mathrm{G}}$ for the Gaussian spheres. Larger $v_{\mathrm{G}}$ reduces the reflectance considerably, whereas smaller $v_{\mathrm{G}}$ increases the reflectivity. The sensitivity tests show that the uncertainty of the $v$ used in the power-law distribution to average the computed singlescattering albedos and scattering matrices for different particle sizes is around \pm 0.2 .

\section{CONCLUSIONS}

Absolute spectral modelling of asteroids gives us valuable information on the mineral composition and particle size distribution of the surface regolith. In this paper, we modelled the absolute reflectance spectra of (4) Vesta by using the ray-optics code SIRIS4, and our measurements of the howardite powder spectra.

First, we obtained Vesta's normalized reflectance spectra observed at a $17.4^{\circ}$ phase angle by Reddy (2011), and scaled them to a geometric albedo value of 0.423 at $0.55 \mu \mathrm{m}$. The photometric phase curve showed that when going from the opposition to a $17.4^{\circ}$ phase angle, Vesta's reflectance decreased by a factor of 0.491 at $0.55 \mu \mathrm{m}$. This was then further applied to the spectra to obtain the absolute reflectance spectra of Vesta at the $17.4^{\circ}$ phase angle.

Secondly, we retrieved the complex refractive indices of the howardite powder by utilizing SIRIS4. A sensitivity analysis was performed on the derived refractive indices. Thirdly, we modelled the absolute reflectance spectra of Vesta using particles with sizes ranging from 10 to $200 \mu \mathrm{m}$. Finally, we carried out sensitivity tests for the Gaussian spheres used in the model.

The best match between the modelled spectrum and observed spectra was found by averaging howardite particles with a size range of 10-200 $\mu \mathrm{m}$ over a power-law distribution with a powerlaw index $v=3.2$. This suggests that Vesta's surface regolith is dominated by small howardite particles (more than 75 percent of particles with a grain size $<25 \mu \mathrm{m}$ ). The differences between the modelled spectrum and the observed spectra can be caused by (a) too small $\operatorname{Im}(m)$ due to leaving out wavelength-scale surfaceroughness and/or volume-inhomogeneity effects when retrieving complex refractive indices, (b) a different type of howardite used in the model, (c) presence of other minerals on Vesta's surface, (d) temperature effects, and/or (e) a more elaborate particle size distribution needed to model the spectra. In the future, we plan to carry out detailed studies of the effects caused by the surface roughness and volume inhomogeneities.

Our simulation framework opens a new field, the modelling of absolute reflectance spectra of asteroids and meteorites while simultaneously considering regolith chemistry and morphology. The first results are extremely promising, and we will develop our model further by taking into account wavelength-scale particles, and studying their effect on the modelled spectra.

\section{ACKNOWLEDGEMENTS}

The research was funded in part by the ERC Advanced Grant No. 320773 (SAEMPL). Computational resources were provided by CSC - IT Centre for Science Ltd, Finland.

\section{REFERENCES}

Busarev V. V., 2010, Sol. Syst. Res., 44, 507

Cellino A. et al., 2016, MNRAS, 456, 248 
Cellino A., Zappalà V., Farinella P., Di Martino M., Paolicchi P., 1987, Icarus, 70, 546

Chapman C. R., Gaffey M., McFadden L., 1993, 24-color Asteroid Survey. EAR-A-DBP-3-RDR-24COLOR-V2.1. NASA Planetary Data System

Cohen M., Witteborn F. C., Roush T., Bregman J., Wooden D., 1998, AJ, 115,1671

De Sanctis M. C. et al., 2011, Space Sci. Rev., 163, 329

De Sanctis M. C. et al., 2012, Science, 336, 697

De Sanctis M. C. et al., 2013, Meteorit. Planet. Sci., 48, 2166

Dotto E. et al., 2000, A\&A, 358, 1133

Hapke B., 1993, Theory of Reflectance and Emittance Spectroscopy. Cambridge Univ. Press, Cambridge

Hicks M. D., Buratti B. J. et al., 2014, Icarus, 235, 60

Hiroi T., Pieters C. M., Takeda H., 1994, Meteorit. Planet. Sci., 29, 394

Jaumann R. et al., 2012, Science, 336, 687

Kohout T., Malina O., Penttilä A., Kröger A., Britt D., Filip J., Muinonen K., Zbořil R., 2015, Space Weathering Induced Slope Changes in Pyroxene and Howardite Spectra, Space Weathering of Airless Bodies, Workshop Abstract

Li et al., 2013, Icarus, 226, 1252

Lim L. F., McConnochie T. H., Bell J. F., Hayward T. L., Hayward T. L., 2005, Icarus, 173, 385

Lindqvist H., Martikainen J., Räbinä J., Penttilä A., Muinonen K., 2018, J. Quant. Spectrosc. Radiat. Transfer, 217, 329

Marchi S. et al., 2012, Science, 336, 690

Markkanen J., Väisänen T., Penttilä A., Muinonen K., 2018, Opt. Lett., 43, 2925

Martikainen J., Penttilä A., Gritsevich M., Lindqvist H., Muinonen K., 2018, J. Quant. Spectrosc. Radiat. Transfer, 204, 144

McCord T. B., Adams J. B., Johnson T. V., 1970, Science, 168, 1445
McSween H. Y. et al., 2013, Meteorit. Planet. Sci., 48, 2090

Muinonen K., Nousiainen T., Lindqvist H., Munoz, O, Videen G., 2009, J. Quant. Spectrosc. Radiat. Transfer, 110, 1628

Muinonen K., Markkanen J., Väisänen T., Peltoniemi J., Penttilä A., 2018, Opt. Lett., 43, 683

Penttilä A., Martikainen J., Gritsevich M., Muinonen K., 2018, J. Quant. Spectrosc. Radiat. Transfer, 206, 189

Reddy V. et al., 2012, Icarus, 217, 153

Reddy V. et al., 2012, Science, 336, 700

Reddy V., 2011, Reddy Vesta Rotationally Resolved Near-Infrared Spectra V1.0. EAR-A-I, 0046-3-REDDYVESTA-V1.0. NASA Planetary Data System

Reddy V., Nathues A., Gaffey M. J., 2011, Icarus, 212, 175

Russell C. T. et al., 2012, Science, 336, 684

Schenk P. et al., 2012, Science, 336, 694

Schröder S. E., Mottola S., Keller H. U., Raymond C. A., Russell C. T., 2014, Planet. Space Sci., 103, 66

Scott A., Duley W. W., 1996, ApJ, 105, 401

Tedesco E. F., Noah P. V., Noah M., Price S. D., 2004, IRAS Minor Planet Survey. IRAS-A-FPA-3-RDR-IMPS-V6.0. NASA Planetary Data System

Thomas P. C., Binzel R. P., Gaffey M. J., Storrs A. D., Wells E. N., Zellner B. H., 1997, Science, 277, 1492

Wilkman O., Muinonen K., Peltoniemi J., 2015, Planet. Space Sci., 118, 250

Wilkman O. V., Gritsevich M., Zubko N., Peltoniemi J. I., Muinonen K. O., 2016, J. Quant. Spectrosc. Radiat. Transfer, 185, 37

This paper has been typeset from a $\mathrm{T}_{\mathrm{E}} \mathrm{X} / \mathrm{LT} \mathrm{T} \mathrm{X}$ file prepared by the author. 